\title{
Thermodynamic Sampling of the Configuration Space of Au-Pt Alloy Nano-slabs Using Metropolis Monte Carlo Simulations
}

Nicole Smina, Joshua Schrier*, Christopher Koenigsmann*

Department of Chemistry, Fordham University, 441 East Fordham Road, Bronx, NY 10458

Introduction

This project employs computational models to study the structural arrangement and properties of alloyed a as cohesive energy per atom, surface distribution, and number of pair sites. Specifically, the project generate configurations of other geometries and sizes, and with different elements. The simulation can be run both in vacuum and in an environment of oxygen. For each simulation, we generate a random

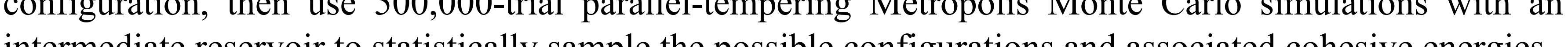
We use a low temperature value of $\mathrm{kT}=0.025 \mathrm{eV}$ to correspond to room temperature, an intermethe reservoir a The simulations allows us to determine, which structural arrangements are statistically most likely to occur, given a speciliedsice,

\section{Experimental Basis}

In a previous project, we synthesized, characterized, and electrochemically tested 50-nm diameter alloy nanowires of various compositions of Au and Pt. Synthesis employs a template-based approach, in which reducing agent sodium borohydride, is poured into the other. $\mathrm{A}$ polycarbonate templet with pores reducing agent, sodium boresponding to the wire diameter size separates the two sides of the tube, and the borohydride reduces
corres metal ions to solid, in the shape of a tubular wire. Characterization via energy-dispersive analysis of $X$ rays (EDAX) and X-ray diffraction (XRD) determines the overall ratio of Au to $\mathrm{Pt}$ in the bulk sample. $\mathrm{X}$ ray Photoelectron Spectroscopy (XPS) is employed to determine the surface composition of the wires and
the influence of the alloy on the electronic properties of the Pt active sites. determines the ratio of Au on of the nanowire.
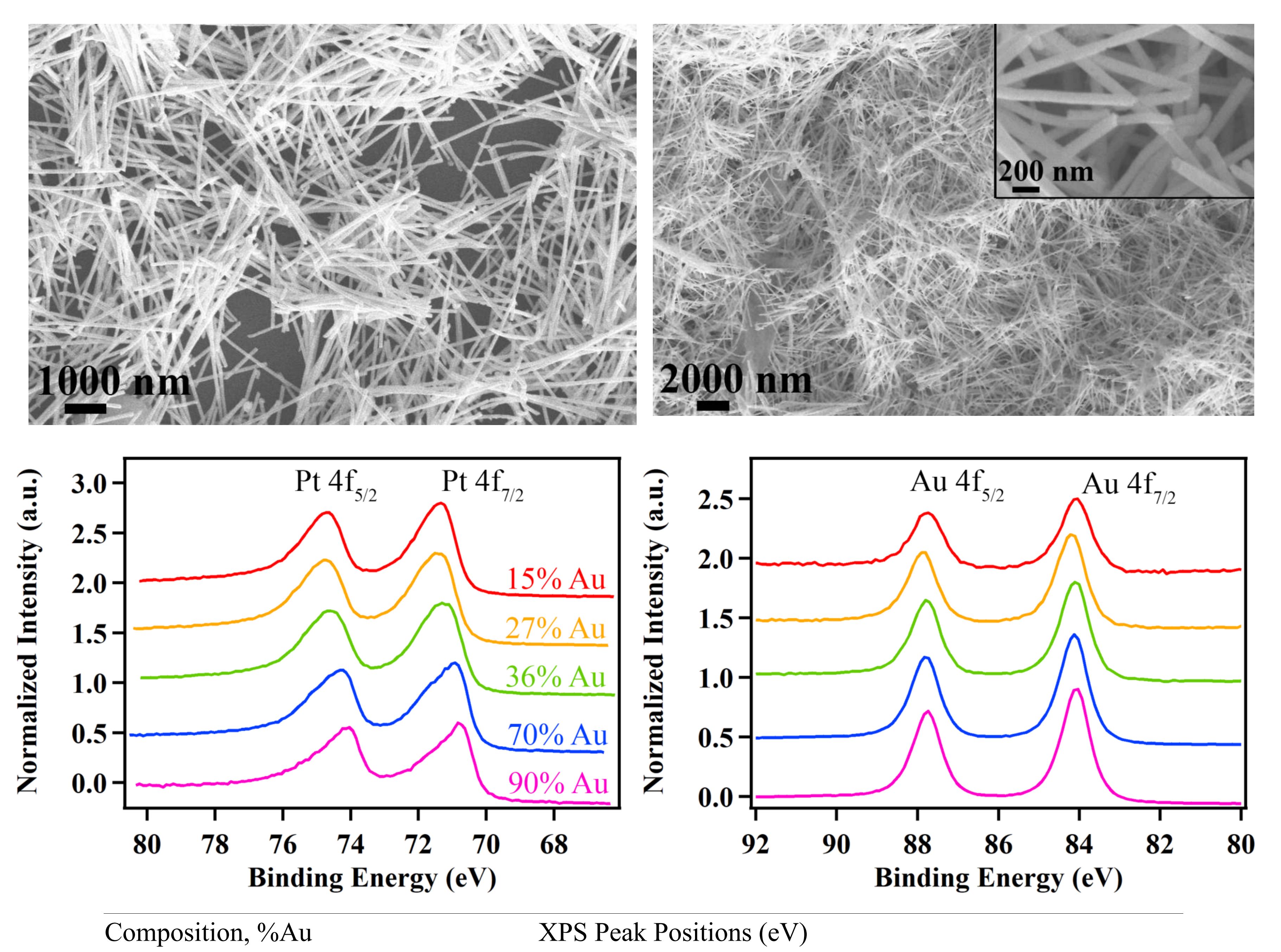

西

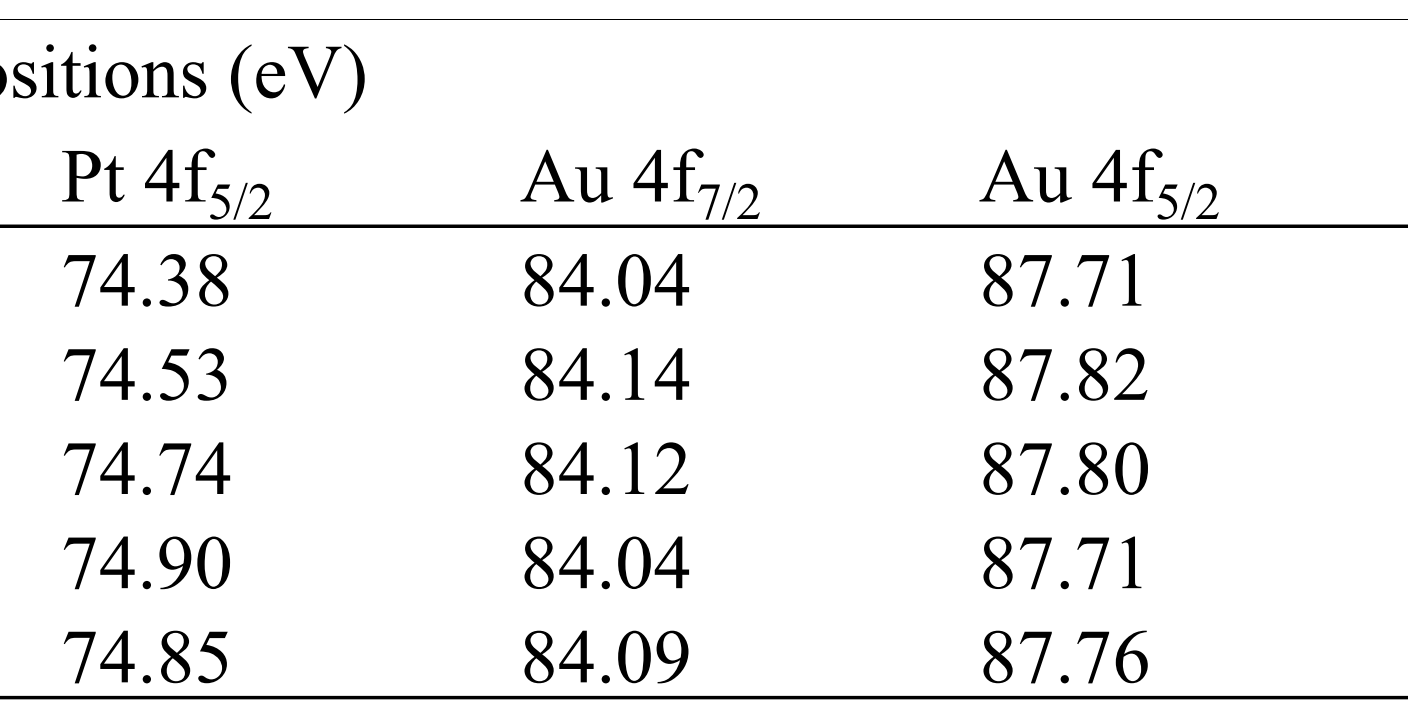

89.9
70.1
35.7
26.9
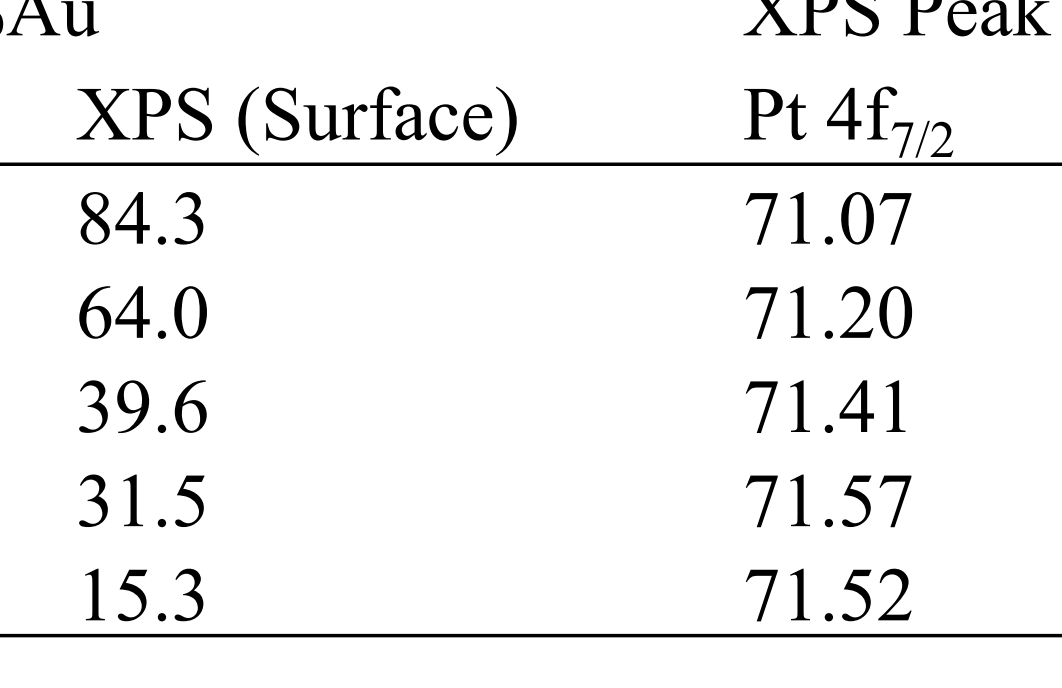

74.90

Nanowire Characterization: SEM images (top) of $P t_{L_{-x}} A u_{x}$ alloy nanowires with compositions of 90\% Pt. (left) and 10\% Pt (right). The XPS spectra (middle) of the Pt and Au $4 f$ peaks as a function of overall composition depict a measurable shift in the Pt 4 f peaks to lower binding
energy with increasing Au content. The XPS data is summarized in the table (bottom). As Pt energy with increasing Au content. The XPS data is summ
content increases, relative fraction of Pt on surface decreases.
Generation of Slabs

The generation of slabs was accomplished via a Python code that takes as input desired size (number Miller index that defines the surface of the slab. Assuming a random distribution of atoms on the facecentered cubic ( $\mathrm{FCC}$ ) sites, the code constructs a slab from an FCC unit cell with a lattice parameter lattice parameter from the lattice parameters of the elements and the nominal composition. The slab can be visualized, color-coded by element. Subsequent calculations can be performed in vacuum or oxygen adsorbates can be added to the surface to simulate In a 2018 paper published in Nanoletters, Yan et. al. developed a model to calculate the cohesive atom depends on the number and identity of atoms with which it neighbors. To be considered nearest neighbors, the distance between two atoms must be smaller than the sum of their van der Waal's radii. A Python code is used to determine the nearest numbers of each atom and to calculate coordination numbers. The cohesive energy is then calculated using the equation

$$
C E=\frac{\sum_{1}^{m} \gamma_{i} \times \frac{C E_{\text {bulk }, i}}{C N_{i}} \sqrt{\frac{C N_{i}}{C B_{i}}}+\gamma_{j} \times \frac{C E_{\text {bulk,j }}}{C N_{j}} \sqrt{\frac{C N_{j}}{C B_{i}}}}{n}
$$

Parallel-Tempering Metropolis Monte Carlo Simulations

\section{Stepwise Summary of Metropolis Monte Carlo Algorithm:}

\section{- Swap an Au atom with a Pt atom}

If new arrangement is lower in energy than original, implement the change $100 \%$ of the time If new arrangement is higher in energy, implement the change only if a random real number between 0
and 1 is less than $\mathrm{e}^{-\Delta \mathrm{E} k \mathrm{~T}}$, where $\Delta \mathrm{E}$ is the energy change, $\mathrm{k}$ is the Boltzmann constant, and $\mathrm{T}$ is the temperature in Kelvin
Repeat simulation over a sufficiently large number of trials to obtain a statistical sampling of Parallel Tempering Algorithm:

- Run three MMC simulations, at low, intermediate, and high temperature - For $10 \%$ of he moves, atchpl to swap the cirrent configuration of the intermediate-temperature simulation with that of the low-temperature simulation, and that of the high-temperature simulation
with that of the intermediate-temperature simulation (the latter to conserve the number of data points collected per reservoir) and use the Metropolis acceptance criteria described above
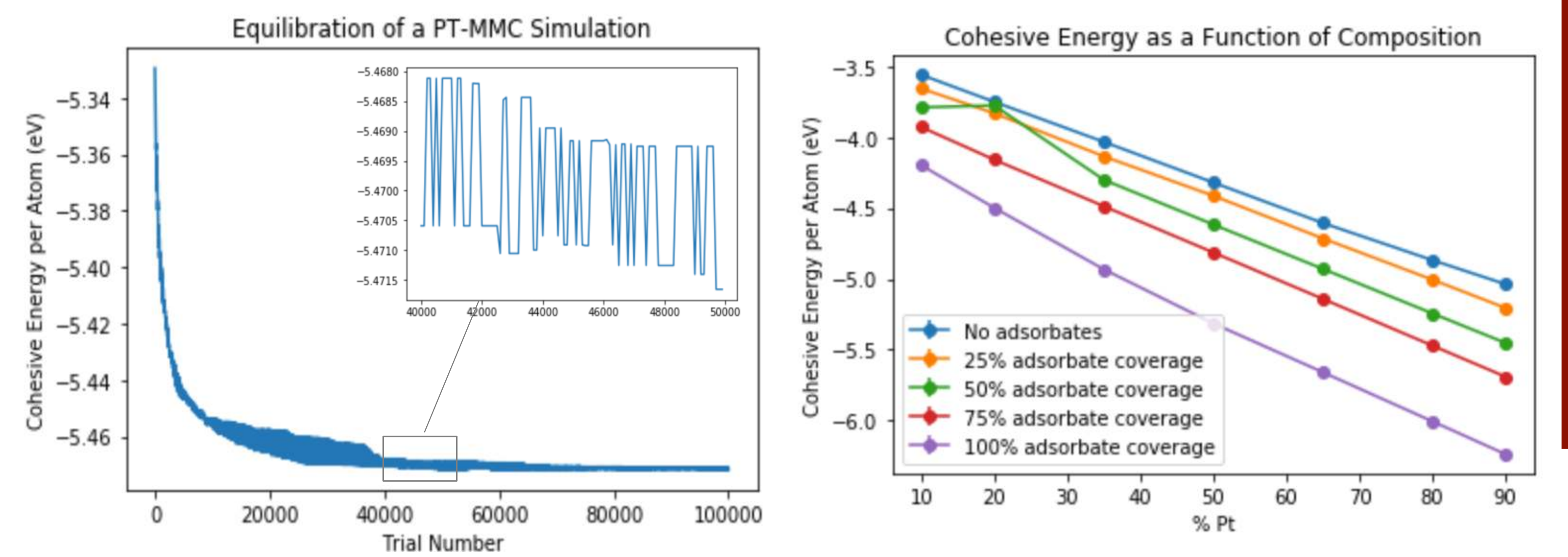

\section{Results}

Simulations are run on 900 -atom slabs across the range of compositions from $10 \%$ Pt to $90 \%$ Pt defined by the (100) Miller index, for which 63\% of total atoms are surface atoms and 37\% are fully-coordinated bulk atoms. Simulations are performed both in vacuum and in oxygen-rich environments in which a specified fraction of
surface atoms contain oxygen adsorbates. Each is a 500,000-trial parallel-tempering Monte Carlo simulation $(\mathrm{kT}$ cold $=0.025 \mathrm{eV}$, $\mathrm{kT}$ intermediate $=0.035 \mathrm{eV}$, kT hot $=0.045 \mathrm{eV}$, swap rate $10 \%$. . The first 100,000 moves for large slabs are discarded as equilibration steps, and every $900^{\text {th }}$ move from
compute averages of energies and properties over the course of the simulation.

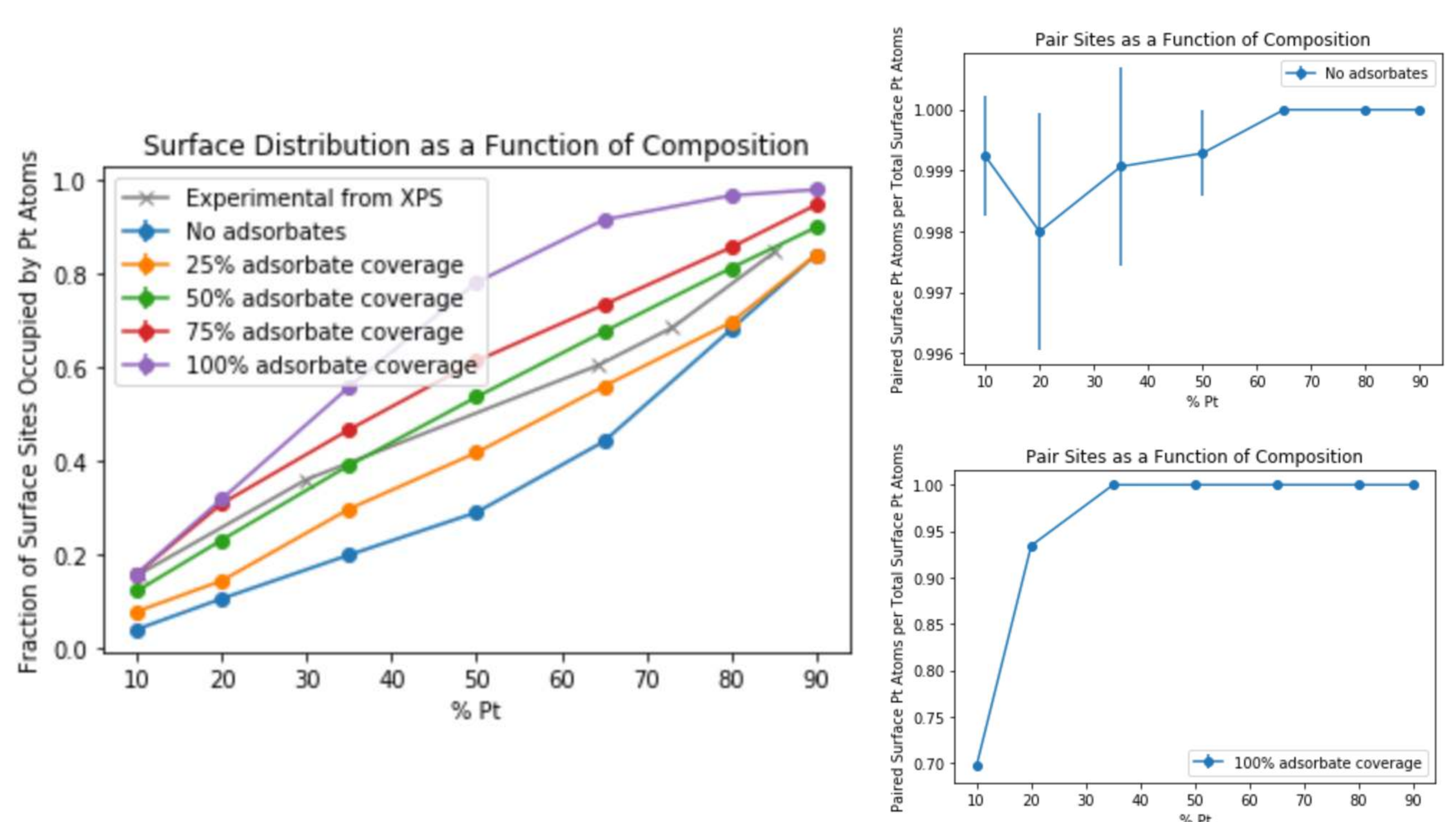

The surface distribution as a function of composition does not match experimental data (predicted by XPS) regardless of the value set for the adsorbate coverage parameter. This suggests that the model, which accouns for enthalpic factors only, is
influence surface distributio

From plot of number of paired $\mathrm{Pt}$ atoms per total Pt atoms on surface, it is apparent that on average, in the absence of adsorbate coverage, each strace $\mathrm{Pt}$ alom tends to be paried. When sichce is covered in adso

dynamic equilibrium.

\section{Acknowledgements}

We would like to thank the National Science Foundation, the Dean of the Fordham College at Rose Hill, and the Clare Boothe
Luce foundation for financial support of this research. Thanks also to William Beatrez, Adam Rosen, and Rosario Troia for their
work on experimental synthesis, and to Michael Tynes and Aaron Dharna for their technical support resolving Python-related

\section{References}

Yan, Z; Taylor, M.G.; Mascareno, A: Mpourmpakis, G. Size, Shape-,
Nanoparticicl Stability Prediction. Nano Letters. 2018, 18 (4), 2696-2704.

Timoshenko, J. et. al. Probing Atomic
Nano Letters 2019, 19, 1, 520-529.

Petkov, V. e e al. Deviations from Vegard's law and evolution of the electrocatalytic activity and stability of Pt-based nanoalloy 